Results The presentations will provide examples of how such models can offer a richer description of epidemiologic associations. Insights may be important when risk assessments are based on epidemiologic results that assess cumulative exposures without consideration of exposure patterns or age-related susceptibility.

Conclusions Models that encompass dynamic aspects of exposure should be encouraged in risk modelling. Such models may also provide information about biologic pathways of disease, leading to better understanding of (for instance) the impact of metabolic saturation on the observed exposure-response curve, or the natural progression of the disease.

\section{DISENTANGLING THE EFFECT OF EXPOSURE DURATION, INTENSITY, AND TIME SINCE EXPOSURE IN STUDIES OF CHRONIC HEALTH EFFECTS}

Jelle Vlaanderen, Lutzen Portengen, Roel Vermeulen. IRAS Utrecht University, Utrecht, The Netherlands

10.1136/oemed-2014-102362.384

Objectives Due to their interrelatedness, modelling independent effects of intensity, duration, and time since exposure on disease risk is complex. The indiscriminate use of the cumulative exposure metric (product of intensity and duration of exposure) might bias reported associations between exposure to hazardous agents and disease risk. We explored the use of a general framework to flexibly model the effects of intensity, duration, and time since exposure on chronic disease.

Method We will provide examples of models falling within the flexible framework. One of such models is an excess relative risk model that is linear in cumulative exposure and exponential in the intensity (or duration) of exposure and time since cessation. This model has been applied successfully to explore effect modification of cumulative exposure by intensity (or duration) of exposure for a number of exposures. We will demonstrate the application of this model in two studies of smoking and chronic disease.

Results In our example the excess relative risk model generally fits the data best. In both studies we observed a strong effect of time since cessation. We observed effect modification by intensity of smoking in one study.

Conclusions Application of flexible models will provide insight into whether the use of cumulative exposure in an epidemiological analysis is justified or whether reducing complex exposure history to a metric such as cumulative exposure is overly restrictive. Combining information on observed patterns of effect modification with mechanistic insights might contribute to the incorporation of biological hypotheses in the development of more biologically relevant exposure metrics.

\section{PROTRACTING EXPOSURES}

David Richardson. Department of Epidemiology, Chapel Hill, NC, USA

10.1136/oemed-2014-102362.385

Objectives When an exposure is protracted or repeated over time, questions arise regarding variation in the effect for different temporal patterns of exposure. We review approaches to describe variation in an exposure's effect as a function of age-atexposure, time-since-exposure, and exposure rate. These models implicitly assume that the effect of an exposure increment on subsequent disease risk depends upon its intensity and timesince-exposure, but not upon the intensities of prior exposures.
Then, we consider the possibility that the effect of an exposure is dependent upon earlier exposures. We use the term 'desensitisation' to refer to the scenario in which a person's response to an exposure diminishes if they have been previously exposed to it. We use the term 'sensitisation' to refer to the scenario in which a person becomes more susceptible to the effect of an exposure if they have been previously exposed to it.

Method We propose a general model for analysis of disease rates in a setting of protracted or repeated exposure that encompasses (de)sensitisation. We illustrate the model using empirical data from a cohort mortality study.

Results The presentation provide examples of how such models can offer a insights into a notion of interaction between an exposure at one point in time and later exposure to the same agent.

Conclusions The possibility that an exposure's effect may depend upon prior exposure to it is often considered in narrative descriptions of etiological processes, yet not readily accommodated by most standard approaches for analysis of protracted occupational and environmental data.

\section{REPRODUCTIVE EFFECTS OF WORKING NIGHT AND ROTATING SHIFTS}

Christina Lawson. The National Institute for Occupational Safety and Health, Cincinnati, Ohio, USA

\subsection{6/oemed-2014-102362.386}

Objectives Recent studies suggest that shift workers who experience exposure to light at night could be at increased risk for adverse reproductive outcomes.

Method Defined by cyclical patterns of circulating hormones, the reproductive system is vulnerable to shifts in circadian rhythms, either through sleep disturbances, altered melatonin production, exposure to light at night, or some other mechanism. Several occupational groups, including health care workers, law enforcement, firefighters, and manufacturing workers are required to work night shifts. Worldwide, millions work at least one night per month.

Results Research will be reviewed on shift work and reproductive outcomes, including menstrual cycle patterns, fertility, pregnancy loss, preterm delivery, and birth weight. The limitations of current research will also be discussed: is there a dose response effect from the number of years of shift work, or can the effects be reversed once shift work stops? Are there different effects from permanent night shift versus rotating shift involving nights?

Conclusions Future research needs will be identified, including the need for validation of self-reported shift work data and the mechanisms by which shift work affects reproductive health. Recommendations for shift workers and employers will be explored.

\section{DYNAMICS OF EXPOSURE AND DISEASE PROGRESSION:} THE USE OF COMPARTMENTAL MODELS

Marc Chadeau-Hyam. Imperial College, London, UK

\subsection{6/oemed-2014-102362.387}

Objectives Chronic diseases are usually slow-developing condition and their risk may result from both long-term exposure and successive exposure increments, hence calling for models accounting for dynamics of exposure and disease progression. 